\title{
Ambiente Contábil
}

\section{REVISTA AMBIENTE CONTÁBIL}

Universidade Federal do Rio Grande do Norte ISSN 2176-9036

Vol. 8. n. 1, jan./jun. 2016

Sítios: http://www.periodicos.ufrn.br/ambiente http://ccsa.ufrn.br/ojs/index.php?.journal=contabil http://www.atena.org.br/revista/ojs-2.2.3-06/index.php/Ambiente

Artigo recebido em: 09.01.2015. Revisado por pares em: 30.03.2015. Resubmetido em: 06.05.2015. Reformulado em: 03.06.2015. Avaliado pelo sistema double blind review.

\section{COMPORTAMENTO DA ARRECADAÇÃO PRÓPRIA E DA CARGA TRIBUTÁRIA NOS MUNICÍPIOS DA ZONA DA MATA MINEIRA}

\section{THE OWN TAX REVENUES AND TAX LOAD PERFORMANCE IN THE MUNICIPALITIES OF THE "ZONA DA MATA MINEIRA"}

\section{COMPORTAMIENTO DE LA RECAUDACIÓN PROPIA Y DE LA CARGA TRIBUTARIA EN LOS MUNICIPIOS DE LA "ZONA DA MATA MINEIRA"}

\section{Autores}

\section{Anderson de Oliveira Reis}

Mestre em Administração pela Universidade Federal de Viçosa (UFV). Professor do Departamento de Ciências Contábeis da Universidade Federal de Juiz de Fora - Campus Avançado de Governador Valadares (UFJF - GV). Avenida Doutor Raimundo Monteiro

Rezende, Centro - Governador Valadares, MG - Brasil. Telefone: (33) 33400430.

E-mail: anderson.reis@ufjf.edu.br

\section{Luiz Antônio Abrantes}

Doutor em Administração pela Universidade Federal de Lavras (UFLA). Professor Associado do Departamento de Administração e Contabilidade da Universidade Federal de Viçosa (UFV). Endereço: Av. PH Rofs, s/n - Campus Universitário. CEP: 36.570-000 - Viçosa/MG Brasil. Telefone: (31) 3899-2886.

E-mail: abrantes@ufv.br.

\section{Walmer Faroni}

Doutor em Administração e Finanças Públicas pela Universitat de Valencia - Espanha. Professor titular do Departamento de Administração e Contabilidade da Universidade Federal de Viçosa (UFV). Endereço: Av. PH Rofs, s/n - Campus Universitário. CEP: 36.570-000 Viçosa/MG - Brasil. Telefone: (31) 3899-2886.

E-mail: w.faroni@ufv.br

\section{Natália Leandro dos Passos}

Graduada em Ciências Contábeis pela União de Ensino Superior de Viçosa (UNIVIÇOSA). Endereço: Rua Gerhardus Lambertus Voorpostel, n . 10, Bairro Liberdade, Viçosa/MG Brasil. CEP: 36570-000 - Telefone: (31) 3899-8000. 
Revista Ambiente Contábil - ISSN 2176-9036 - UFRN - Natal-RN. v. 8. n. 1, p. 1 - 16, jan./jun. 2016.

\title{
RESUMO
}

O processo de descentralização consolidado pela constituição de 1988 mudou a dinâmica do federalismo brasileiro aumentando as receitas disponíveis e também as responsabilidades do fornecimento de serviços para os municípios, que em termos financeiros são muito dependentes das transferências governamentais. Diante da importância dos municípios na Administração Pública como entes federativos, este estudo tem como objetivo identificar, a partir da classificação em grupos, a mudança no perfil da arrecadação tributária própria dos municípios da Zona da Mata Mineira tendo por base os períodos de 2005 e 2010. Trata-se de pesquisa descritiva e quantitativa em que se utilizou as metodologias de Análise de Cluster e Teste t para amostras emparelhadas. Os resultados apontam para formação de 4 grupos de acordo com o esforço fiscal (baixo, médio e alto). Em média observa-se uma evolução positiva na arrecadação própria dos municípios que foi confirmada pelo Teste t. Conclui-se que apesar da dependência dos recursos de transferências, os municípios têm realizados esforços para aumentar a arrecadação própria e consequentemente suas receitas disponíveis.

Palavras-Chave: Finanças Públicas. Receita Tributária. Administração Pública.

\begin{abstract}
The process of decentralization consolidated by "Brazilian Constitution of 1988" changed the dynamics of the Brazilian federalism increasing the available revenues and also the responsibilities of providing services to municipalities, in which, in financial terms, are highly dependent on governmental transfers. Given the importance of the municipalities in the Public Administration as federative entities, this study aims this study aims to identify, from the classification groups, the change in the profile of own tax revenues of municipalities in the "Zona da Mata Mineira" based on the periods of 2005 and 2010. It is a descriptive and quantitative study in which it was used the methodologies of Cluster Analysis and the T-Test for paired samples. The results indicate a formation of four groups according to the fiscal effort (low, medium and high). On average, it was observed a positive development in own tax collection of the municipalities which was confirmed by T-Test. It concludes that despite the dependence on transfers of resources, the municipalities have made efforts to increase their collection and consequently their available revenues.
\end{abstract}

Keywords: Public Finances. Tax Revenue. Public Administration.

\section{RESUMEN}

El proceso de descentralización consolidado por la "Constitución Brasileña de 1988" cambió la dinámica del federalismo brasileño con el aumento de los ingresos disponibles y también las responsabilidades de la prestación de servicios a los municipios, que son económicamente muy dependientes de las transferencias del gobierno. Dada la importancia de los municipios en la administración pública como entes federales, el presente estudio objetiva identificar, a partir de los grupos de clasificación, el cambio en el perfil de los ingresos tributarios propios de los municipios de la "Zona da Mata Mineira" entre el período de 2005 y 2010. Es una investigación descriptiva y cuantitativa en la cual se utilizó la metodología de Análisis Cluster y Test $\mathrm{T}$ para las muestras apareadas. Los resultados muestran a la formación de cuatro grupos de acuerdo con el esfuerzo fiscal (bajo, medio y alto). En promedio, se observa una evolución positiva en la recaudación propia de los municipios que se confirmó con el Test T. Se concluye que a pesar de la dependencia de las transferencias de recursos, los municipios han hecho esfuerzos con el objetivo de aumentar su recaudación propia y por lo tanto sus ingresos disponibles.

Palabras clave: Finanzas Públicas. La Recaudación Tributaria. Administración Pública. 
Revista Ambiente Contábil - ISSN 2176-9036 - UFRN - Natal-RN. v. 8. n. 1, p. 1 - 16, jan./jun. 2016.

\section{INTRODUÇÃO}

O processo de descentralização verificado após a Constituição de 1988 criou um desafio para o arranjo do pacto federativo brasileiro. Os municípios receberam maiores atribuições e passaram a ter maior autonomia financeira, com competência tributária própria. Além disso, tiveram um aumento de recursos, via transferências governamentais da União e Estado, para a provisão de serviços públicos e implementação das políticas públicas.

Para Afonso e Araújo (2001) a descentralização dos recursos tributários, pode ser considerada como um movimento de municipalização da receita que embora tenha acontecido de forma não adequadamente planejada, resultou em um processo desordenado de descentralização de encargos. Na visão de Reis, Costa e Silveira (2013) os municípios foram os principais beneficiários da descentralização fiscal considerando a atribuição e competências tributárias próprias e ampliação das participações no produto da arrecadação de impostos federais e estaduais, via transferências.

Entretanto, alguns fatores como a manutenção da eficiência do sistema tributário, a redução dos custos incorridos pelos contribuintes no cumprimento da obrigação tributária e melhor aplicação dos recursos, o produto da arrecadação manteve-se centralizada. Para Mendes et al. (2008) a centralização da arrecadação gera um desequilíbrio vertical, considerando que os poucos tributos que podem ser arrecadados com eficiência nos estados e municípios não são suficientes para custear os gastos desses níveis de governo. Uma forma de solucionar o problema é manter a arrecadação centralizada e fazer transferência de recursos para os governos subnacionais.

Estas transferências ameniza o desequilíbrio existente no processo arrecadatório, em algumas regiões de base econômica e tributária mais frágil e permite reduzir as desigualdades sociais e regionais, sendo para Rezende (2006) um dos desafios do federalismo fiscal brasileiro.

Na visão de Afonso e Araújo (2001) é um erro acreditar que todo governo deveria ser autossustentável, mesmo aqueles de menores escala e em regiões mais pobres, pois os tributos típicos de governos locais - especialmente incidentes sobre o patrimônio e taxas - são os que apresentam maior dificuldade para se administrar e explorar seu potencial. Para Baião et. al. (2014), a literatura aponta a importância das transferências para atenuar as desigualdades regionais através da equalização fiscal, beneficiando municípios com menor base tributária e maiores custos de provisão dos serviços públicos. Essas transferências buscam tornar a provisão dos serviços públicos mais equitativa, permitindo que os governos locais, para dado nível de esforço fiscal, ofereçam o mesmo volume e qualidade de serviços à população.

As transferências constitucionais representam a maior fonte de receita orçamentária para os municípios que muitas das vezes se tornam dependentes delas. Entretanto muitos municípios conseguem incrementar a arrecadação própria imprimindo maior rigor na fiscalização e cobrança dos tributos e usando a tecnologia para aprimorar o processo. Diante deste contexto, considerando o papel dos municípios para a Administração pública brasileira e em contraponto a sua fragilidade de gestão devido a aspectos de capacidade técnica e política, uma questão é levantada: A participação das receitas próprias na totalidade da arrecadação tributária tem evoluído positivamente ao longo dos anos?

Dessa forma, esta pesquisa visa identificar a mudança no perfil da arrecadação tributária própria dos municípios da Zona da Mata Mineira. Especificamente pretende-se i) identificar e analisar o percentual de participação das receitas tributárias, na arrecadação total dos municípios; ii) classificar os municípios conforme a homogeneidade dos grupos a serem 
Revista Ambiente Contábil - ISSN 2176-9036 - UFRN - Natal-RN. v. 8. n. 1, p. 1 - 16, jan./jun. 2016.

formados com base nos indicadores de receita orçamentária estabelecidos e iii) verificar a evolução no esforço de arrecadação entre os períodos de 2005 e 2010.

O marco temporal foi definido com base na Lei de Responsabilidade Fiscal, correspondendo a 5 e 10 anos após a sua vigência, sendo os dados analisados referentes ao período de 2005 e 2010. A justificativa do estudo encontra-se na importância dos municípios em atender as demandas da sociedade por ser o ente federativo mais próximo e, portanto, com maior capacidade de percepção de suas verdadeiras necessidades. Sendo assim, o comportamento da arrecadação própria e da carga tributária pode contribuir para o entendimento da dinâmica de financiamento das políticas públicas locais.

\section{REVISÃO DE LITERATURA}

Esta seção será dividida em duas partes. A primeira versará sobre o federalismo fiscal e a descentralização buscando mostrar suas implicações para o município. Na segunda parte é tratada a composição da receita municipal destacando suas principais características.

\subsection{FEDERALISMO FISCAL E DESCENTRALIZAÇÃO}

O federalismo fiscal trata de uma das questões centrais em qualquer regime federativo que consiste na repartição dos recursos fiscais entre os entes federados de modo que todos disponham de uma capacidade de financiamento compatível com as respectivas responsabilidades (REZENDE, 2010).

O ponto chave do novo federalismo estabelecido pela Constituição de 1988 foi à descentralização. $O$ processo não significava somente o repasse de maior volume de recursos e poder aos governos subnacionais, mas, principalmente, tinha como palavra de ordem a municipalização. Nesse sentido, o Brasil se tornou uma das pouquíssimas federações do mundo a dar status de ente federativo aos municípios (ABRUCIO, 2010).

O equilíbrio entre responsabilidades e recursos em uma federação demanda soluções tão mais complexas quanto maiores forem às disparidades regionais e sociais. Dificuldades para implementar um modelo de federalismo fiscal que trate adequadamente essas disparidades têm estado presentes na história do federalismo brasileiro desde os primórdios de sua adoção (REZENDE, 2010).

Abrucio (2010) esclarece que o processo de descentralização brasileiro foi realizado preferencialmente em prol da municipalização das políticas. No entanto, mantinha-se uma preocupação com a interdependência federativa, na forma de medidas de combate à desigualdade, de preocupações em torno da cooperação intergovernamental e da definição de um raio importante de ações federais como agente nacional.

A estruturação de um federalismo mais equilibrado não é uma meta importante somente para o Brasil, mas em todos os países federativos do mundo. No entanto, na realidade brasileira essa tensão assume características específicas devido, principalmente, as graves desigualdades sociais e regionais existentes no país (LINHARES, MENDES e LASSANCE, 2012).

Gomes e Mac Dowell (2000) chamam a atenção para dois aspectos importantes do processo de descentralização, a intensa criação de municípios e o aumento das receitas postas à disposição dos municípios que apresentam consequências indesejáveis, tanto do ponto de vista econômico quanto do social.

Oliveira, Barbosa e França (2013) destacam que com a Reforma do Estado, ocorreu uma expressiva descentralização de recursos da União para as esferas subnacionais, tanto em termos de arrecadação própria, quanto de receita disponível, sobretudo para os municípios. Neste sentido, O Brasil adota sistematicamente, uma política de redistribuição de recursos 
Revista Ambiente Contábil - ISSN 2176-9036 - UFRN - Natal-RN. v. 8. n. 1, p. 1 - 16, jan./jun. 2016.

através de transferências baseadas em programas focalizados, ficando os estados e municípios obrigados a absorvê-los devido à falta autonomia política e legal para executá-los por si próprios.

Apesar de muito discutida o aumento das receitas disponíveis ao município, que é o principal fator da tensão do federalismo fiscal brasileiro, não gerou os impactos esperados com relação à autonomia desses entes e a redução das desigualdades sociais e regionais.

O município, por ser o ente federativo mais próximo da sociedade e, portanto, mais sensível as suas necessidades, tem potencial de ação para redução das desigualdades. No entanto, como as políticas públicas seguem o modelo top down sem a participação dos municípios em sua formulação, esse potencial se perde em meio as transações federativas e o que se observa é a imposição de políticas da União para a execução em nível local.

Cabe destacar que com o processo de descentralização atribuiu-se mais responsabilidade ao município provendo-o de autonomia frente aos outros entes federativos. No entanto, essa autonomia legitima e autoriza, no momento em que também viabiliza, sua atuação em novas áreas que antes não constavam no rol de atribuições dos administradores municipais (ALCÂNTARA, 2011).

Masardi e Abrantes (2014) destacam que no processo de descentralização houve a criação de relevantes mecanismos distributivos, como as transferências intergovernamentais. Os municípios passaram a concentrar maior parcela de recursos, e concomitantemente, absorveram atribuições adicionais que antes eram da União, resultando em maior participação municipal nos gastos públicos, principalmente naqueles relacionados à educação e à saúde.

Reis, Costa e Teixeira (2013) ressaltam que o processo de descentralização ocorrido no Brasil teve como objetivo ampliar a autonomia administrativa, política e financeira dos municípios, uma vez que estes passaram a ser tratados e reconhecidos como entes federativos. Em contrapartida, os municípios tiveram aumento significativo nas obrigações de prestação de serviços públicos essenciais, que passaram a ser de sua responsabilidade (SOUZA, 2003).

$\mathrm{Na}$ tentativa de corrigir distorções da estrutura anterior, a Constituição Federal de 1988 buscou resgatar o princípio do federalismo fiscal, tentando modernizar a estrutura tributária, além de torná-la progressiva e mais justa, permitindo maior autonomia e aumentando a capacidade de arrecadação dos entes federativos (MATIAS-PEREIRA, 2009).

Serra e Afonso (1999) ressaltam que o fortalecimento e a consolidação da capacidade de tributação dos entes federados são consequência da adoção do federalismo fiscal, além de movimentos no sentido de redistribuição dos recursos públicos, almejando amenizar as disparidades regionais e promover o desenvolvimento de forma mais igualitária do país como um todo.

\subsection{COMPOSIÇÃO DA RECEITA PÚBLICA MUNICIPAL}

Com a descentralização das receitas fiscais os municípios foram os principais beneficiários da descentralização fiscal com a atribuição de competências tributárias próprias e ampliação das participações no produto da arrecadação de impostos federais e estaduais (REIS; COSTA; SILVEIRA, 2013).

A receita municipal é formada pela arrecadação tributária própria e transferências intergovernamentais. Os meios de repasse de receitas de transferências referem-se à Quotaparte do ICMS, ao Fundo de Participação dos municípios (FPM), ao Fundo do IPIExportação (FPEEX), Imposto Territorial Rural (ITR), ao Fundo de Manutenção e Desenvolvimento da Educação Básica (FUNDEB) (ANDRADE, 2010).

A arrecadação própria ou receita tributária municipal é composta por: Imposto sobre a Propriedade Predial e Territorial Urbana (IPTU), Imposto sobre Transmissão de Bens Imóveis (ITBI), Imposto Sobre Serviço de Qualquer Natureza (ISS), contribuições de 
Revista Ambiente Contábil - ISSN 2176-9036 - UFRN - Natal-RN. v. 8. n. 1, p. 1 - 16, jan./jun. 2016.

melhorias e taxas que possam vir a ser cobradas pela administração pública municipal (KOHAMA, 2012).

Gouvêia et al., (2010) consideram que o Fundo de Participação Municipal (FPM), transferência da União para os municípios e a quota-parte do Imposto sobre Circulação de Mercadorias e Serviços (ICMS), repasse estadual, são as principais transferências feitas aos municípios.

Para os municípios são repassados $25 \%$ do total arrecadado pelo Estado, conforme os critérios de transferência da quota-parte de ICMS, sendo a intensidade da produção econômica um dos principais determinantes do volume de repasse. Sendo assim, os repasses dependem da capacidade de geração de riqueza no âmbito municipal. Isso significa que os municípios menos desenvolvidos economicamente não são beneficiados com esses repasses (REIS, COSTA e SILVEIRA, 2013).

$\mathrm{Na}$ percepção de Gouvêa et al. (2010) apesar de os municípios apresentarem aumento na sua capacidade fiscal, esse processo não é observado de forma homogênea entre os municípios. De fato, observa-se que os tributos de competência municipal possuem maior potencial de arrecadação nos médios e grandes municípios, já que nos de pequeno porte, com base predominantemente rural, a propriedade urbana é pouco significativa e, o setor de serviços, pouco expressivo e ainda a de se considerar fatores políticos na gestão tributária (BOVO, 2001).

O processo de descentralização não distribuiu seus benefícios de forma equitativa. A realidade dos municípios de menor porte é a pouca capacidade de expandir a arrecadação de impostos e de financiar outra atividade que não seja o pagamento dos servidores públicos. Dessa forma, não há como desempenhar, com recursos transferidos para tal fim, algumas atividades relacionadas à prestação de serviços de saúde e educação (REIS; COSTA; SILVEIRA, 2013).

Segundo Tomio (2005) há ainda municípios que são incapazes de sustentar até mesmo sua folha de pagamento apenas com a receita própria, criando situação de dependência das transferências do Estado e da União, principalmente do Fundo de Participação Municipal (FPM), que é a maior fonte de renda dos municípios de pequeno porte.

\section{PROCEDIMENTOS METODOLÓGICOS}

Este estudo pode ser caracterizado como descritivo uma vez que busca delinear o comportamento da arrecadação própria e da carga tributária dos municípios da Zona da Mata Mineira. Quanto à forma de abordagem do problema trata-se de uma pesquisa quantitativa, visto a utilização de métodos estatísticos para análise e tratamento dos dados.

A Zona da Mata Mineira, localizada no sudeste de Minas Gerais, com uma área de $36.058 \mathrm{~km}^{2}$ é formada por oito microrregiões: Cataguases, Juiz de Fora, Manhuaçu, Muriaé, Ponte Nova, Ubá e Viçosa, totalizando 142 municípios. A base da sua economia está concentrada na atividade agropecuária, industrial e com grande potencial para o turismo. A escolha dessa região se deve ao fato de, assim como o estado de Minas Gerais, ela é caracterizada, em sua maior parte por municípios de pequeno porte com população entre 1.558 e 46.284 habitantes além de sintetizar as desigualdades socioeconômicas do País.

Os dados secundários foram coletados no relatório de Finanças do Brasil - FINBRA, publicado anualmente pela Secretaria do Tesouro Nacional, que traz informações sobre os orçamentos dos municípios. Nessa base de dados foi possível coletar as receitas de arrecadação própria e a receita orçamentária total por município nos períodos de 2005 e 2010. Os dados sobre a população foram coletados junto a base de dados do Índice Mineiro de Responsabilidade Social (IMRS) divulgado pela Fundação João Pinheiro.

Para alcançar os objetivos deste estudo, inicialmente foi necessária a determinação da participação da arrecadação de impostos na receita total do município. Para isso, foi adaptado 
Revista Ambiente Contábil - ISSN 2176-9036 - UFRN - Natal-RN. v. 8. n. 1, p. 1 - 16, jan./jun. 2016.

um indicador de estrutura de receita definido por Matias e Campello (2000, p.242), obtido a partir da seguinte fórmula:

Participação das Receitas Próprias (PRP) $=\frac{\text { Receitas Próprias }}{\text { Receita Orçamentária Total }}$

Este indicador permite determinar o perfil das receitas tributárias indicando o grau de autonomia dos municípios em relação a outras esferas do governo.

Utilizou-se também um indicador per capita adaptado de KOHAMA (1999), para analisar a carga tributária dos impostos nos municípios, que será obtido a partir da seguinte fórmula:

Carga Tributária dos Impostos Municipais (CTIM) $=\frac{\text { Receitas Próprias }}{\text { População Total }}$

Para atualizar os valores de 2005 em relação a 2010 e para minimizar o efeito da inflação na arrecadação dos municípios da Zona da Mata, com base no Índice de Preço ao Consumidor (IPCA), utilizou-se a seguinte fórmula:

Deflator_ano_t $=\frac{\text { IPCA do ano } \mathrm{x}}{\text { IPCA do ano } \mathrm{t}}$

O tratamento dos dados aconteceu em três etapas. A primeira, consistiu na utilização da análise exploratória dos dados objetivando conhecer o comportamento das variáveis PRP e CTIM com relação às medidas de centro, de dispersão, distribuição e outliers.

$\mathrm{Na}$ segunda etapa, utilizou-se o método estatístico de Análise de Cluster para classificar os municípios conforme a homogeneidade dos grupos a serem formados com base nos indicadores de receita próprias estabelecidos. Este método pode ser definido como "uma das técnicas de análises multivariada cujo propósito é reunir objetos, baseando-se nas características dos mesmos" (CORRAR, PAULO e DIAS FILHO, 2007, p.325).

Assim, os municípios foram classificados em grupos com homogeneidade interna e heterogeneidade externa, sendo similares entre si. A classificação serviu para mostrar que muitas vezes municípios próximos geograficamente podem ser diferenciados em relação à receita tributária própria arrecadada.

A terceira etapa, considerando o intuito de verificar se houve mudança na gestão desses indicadores entre os períodos de 2005 e 2010 foi utilizado o teste de média para amostras emparelhadas. Para utilização deste teste foram constituídas as seguintes hipóteses:

$H_{0:}$ Não existe diferença na média da Participação dos Impostos na Receita Orçamentária nos períodos de 2005 e 2010.

$H_{A}$ : Existe diferença na média da Participação dos Impostos na Receita Orçamentária nos períodos de 2005 e 2010.

$H_{0}$ : Não existe diferença na média da Carga Tributária nos períodos de 2005 e 2010.

$H_{A}$ : Existe diferença na média da Carga Tributária nos períodos de 2005 e 2010. 
Revista Ambiente Contábil - ISSN 2176-9036 - UFRN - Natal-RN. v. 8. n. 1, p. 1 - 16, jan./jun. 2016.

\section{ANÁLISE E DISCUSSÃO DOS RESULTADOS}

Para a realização da pesquisa foram excluídos dos anos de 2005 e 2010 os municípios de Abre Campo, Chalé, Mar de Espanha, Rio Novo, Santa Rita de Jacutinga, São Pedro dos Ferros e Vieiras, por não apresentarem dados suficientes para análise.

Os municípios de Belmiro Braga, Chiador, Juiz de Fora, Oliveira Fortes, Rio Doce, Simão Pereira e Ubá foram também retirados por se apresentarem como outliers durante a realização da análise exploratória dos dados. Assim a pesquisa foi realizada com128 municípios da Zona da Mata, com análise das variáveis participação da receita de impostos e carga tributária per capita.

\subsection{ANÁLISE EXPLORATÓRIA DOS DADOS (AED)}

$\mathrm{Na}$ primeira parte do tratamento dos dados foi realizada a análise exploratória para identificar e avaliar o comportamento das variáveis. A Tabela 1 apresenta a estatísticas descritivas das variáveis participação das receitas próprias (PRP) e carga tributária per capita de impostos municipais (CTIM) para os períodos analisados.

Tabela 1 - Estatística descritiva das variáveis

\begin{tabular}{ccccc}
\hline & PRP 2005 $(\%)$ & CTIM 2005 (R\$) & PRP 2010 (\%) & CTIM 2010 (R\$) \\
\hline Média & 2,79 & 30,69 & 3,62 & 58,74 \\
\hline Mediana & 2,10 & 23,63 & 2,69 & 43,42 \\
\hline Moda & 0,62 & 9,57 & 0,72 & 16,87 \\
\hline Desvio padrão & 1,95 & 20,25 & 2,77 & 47,79 \\
\hline Variância & 3,81 & 410,02 & 7,69 & 2284,13 \\
\hline Mínimo & 0,61 & 9,57 & 0,72 & 16,87 \\
\hline Máximo & 8,60 & 113,53 & 16,55 & 371,14 \\
\hline
\end{tabular}

Fonte: Dados da Pesquisa.

Verifica-se que a participações de impostos na receita orçamentária dos municípios variou de $0,61 \%$ a $8,60 \%$ em 2005 e de $0,72 \%$ a $16,55 \%$ em 2010 , com média de $2,79 \%$ em 2005 e de $3,62 \%$ em 2010. O aumento da média, sem o efeito da inflação, pode representar um empenho dos municípios para arrecadar seus impostos, via redução da inadimplência dos contribuintes ou na busca de alternativas para melhorar a eficiência na arrecadação.

O município que apresentou em 2005 o menor percentual de PRP foi Aracitaba com 0,61\%, enquanto Matias Barbosa apresentou o valor máximo de 8,61\%. Em 2010 o município que apresentou o menor valor de PRP foi Rosário da Limeira, $0,72 \%$ e Santo Antônio do Grama o valor máximo de $16,55 \%$.

Os valores de variância e desvio padrão revelam que não há diferenças consideráveis entre os municípios, que em sua maioria tem baixa participação das receitas próprias na receita disponível, indicando maior dependência das transferências intergovernamentais.

A Carga Tributária per capita variou de $\mathrm{R} \$ 9,57$ a $\mathrm{R} \$ 113,53$ em 2005 e de $\mathrm{R} \$ 16,87$ a $\mathrm{R} \$ 371,14$ em 2010. Com uma média de $\mathrm{R} \$ 30,69$ em 2005 e $\mathrm{R} \$ 58,74$ em 2010, destaca-se a amplitude da amostra nos dois períodos, identificado a existência de municípios com pequenas, médias e grandes cargas tributárias per capita. O município de Cipotânea apresentou o menor valor de $\mathrm{R} \$ 9,57$ e Matias Barbosa em 2005, o valor máximo de $\mathrm{R} \$ 113,53$. Em 2010 o município de Rosário da Limeira apresentou o menor valor de $\mathrm{R} \$ 16,87$ e Santo Antônio do Grama o valor máximo de R $\$ 371,14$.

A carga tributária aumentou nos períodos analisados indicando o aumento na contribuição individual em relação aos tributos. Este indicador apresentou maior desvio 
Revista Ambiente Contábil - ISSN 2176-9036 - UFRN - Natal-RN. v. 8. n. 1, p. 1 - 16, jan./jun. 2016.

padrão em ambos os períodos, com discrepância entre os valores mínimos e máximos, conforme ilustrado na Tabela 1.

\subsection{ANÁLISE DE CLUSTER}

$\mathrm{Na}$ análise de Cluster utilizou-se como variáveis a participação das receitas de impostos e a carga tributária de impostos per capita. Com o objetivo de minimizar a dispersão dentro dos grupos e considerando a inexistência de regras quanto à formação do número de grupos na Análise de Cluster, utilizou-se o agrupamento por K- média optando-se por 4 grupos em ambos os períodos analisados. Os agrupamentos foram obtidos utilizando o programa Statistical Package for the Social Sciences - SPSS versão 21.0.

A Tabela 2 ilustra a distribuição de frequência gerada pela Análise de Cluster no ano de 2005 em que a maior parte dos municípios se concentra no Cluster 1 (58,59\%), seguido pelo Cluster $3(28,90 \%), 4(9,38 \%)$ e $2(3,13 \%)$. Os agrupamentos formados podem ser divididos em Clusters pequenos ( 2 e 4 ) e grandes ( 1 e 3 ).

Tabela 2 - Número de observação por Cluster em 2005

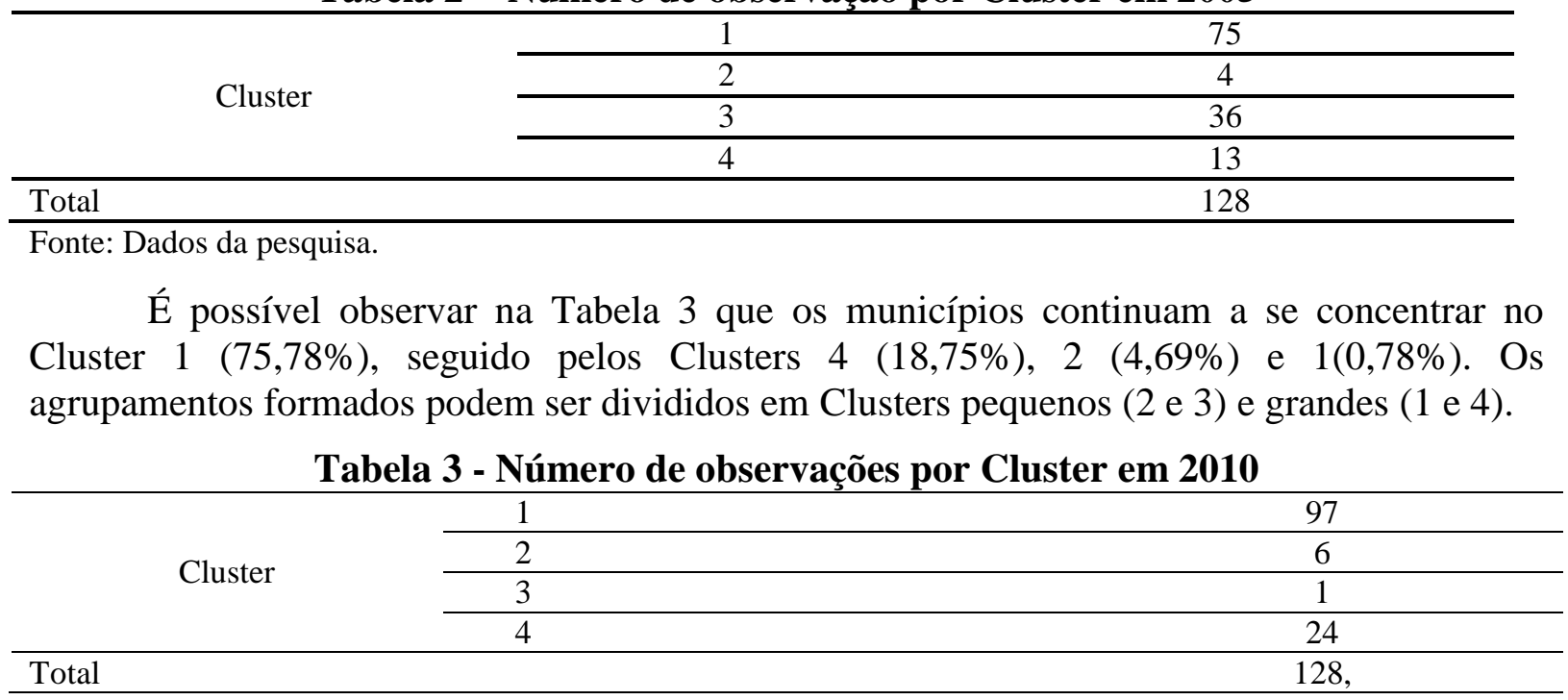

Fonte: Dados da pesquisa.

A mudança na composição dos Clusters evidencia alteração no comportamento da arrecadação própria e na carga tributária entre os períodos analisados indicando maior aglomeração entre os municípios podendo inferir a existência de comportamento igualitário com relação à gestão tributária.

\subsubsection{CLUSTER 1: BAIXA ARRECADAÇÃO PRÓPRIA E CARGA TRIBUTÁRIA}

O Cluster 1 agrupou os municípios com baixa arrecadação própria e baixa carga tributária, caracterizando municípios com maior dependência das transferências governamentais e que aparentemente desenvolvem poucas ações na promoção de melhoria da gestão tributária via aumento da arrecadação própria.

Este grupo contém 75 observações em 2005 (58,59\% do total de municípios examinados) ampliando para 97 observações (75,78\% do total de municípios examinados) em 2010. Esta ampliação pode indicar possível queda na qualidade da gestão tributária da arrecadação própria.

Esse Cluster não é composto pelos mesmos municípios nos dois períodos de análise, 68 municípios (90,67\% das observações de 2005) permaneceram em ambos os períodos, 06 municípios (8\% das observações de 2005) passaram a compor o Cluster 4 no ano de 2010 a exemplo de Faria Lemos, Laranjal, Pedra Bonita, Pedro Teixeira, Piedade de Ponte Nova e 
Revista Ambiente Contábil - ISSN 2176-9036 - UFRN - Natal-RN. v. 8. n. 1, p. 1 - 16, jan./jun. 2016.

Tombos. Apenas município de Sem-Peixe (1,33\% das observações de 2005) passou a integrar o Cluster 2.

Em 2010 o cluster 1 teve 29 municípios a mais do que em 2005, sendo eles: Acaiaca, Alto Caparaó, Antônio Prado de Minas, Astolfo Dutra, Caparaó, Carangola, Chácara, Dom Silvério, Ervália, Guarani, Guarará, Lajinha, Lamim, Lima Duarte, Manhumirim, Mirai, Paiva, Piau, Raul Soares, Recreio, Rio Casca, Rio Pomba, Rochedo de Minas, Santa Bárbara do Monte Verde, São Francisco do Glória, Senador Cortes, Silverânia, Tabuleiro, Tocantins.

A estatística descritiva do Cluster 1 para o ano de 2005 e 2010, apresentada na Tabela 4, permite identificar que a participações dos tributos dos municípios do Cluster 1 no ano de 2005 variou de $0,62 \%$ a 3,27\%, com média de 1,77\%. O indicador carga tributária de impostos per capita variou de $\mathrm{R} \$ 9,57$ a $\mathrm{R} \$ 26,82$ no ano de 2005 com média de $\mathrm{R} \$ 18,50$.

Tabela 4 - Análise descritiva Cluster 1

\begin{tabular}{ccccc}
\hline & PRP2005(\%) & PRP2010 (\%) & CTIM2005(R\$) & CTIM2010 (R\$) \\
\hline Média & 1,77 & 2,56 & 18,50 & 39,01 \\
\hline Mediana & 1,65 & 2,23 & 18,47 & 38,35 \\
\hline Moda & 0,62 & 0,72 & 9,57 & 16,87 \\
\hline Desvio padrão & 0,62 & 1,15 & 4,61 & 13,55 \\
\hline Variância & 0,39 & 1,33 & 21,27 & 183,52 \\
\hline Mínimo & 0,62 & 0,72 & 9,57 & 16,87 \\
\hline Máximo & 3,27 & 6,44 & 26,82 & 66,67 \\
\hline
\end{tabular}

Fonte: Dados da Pesquisa.

Em 2010, a participação da receita de impostos variou de $0,72 \%$ a 6,44\%, com média de 2,56\% da receita total dos municípios. A carga tributária de impostos municipais variou de $\mathrm{R} \$ 16,87$ a $\mathrm{R} \$ 66,67$, alcançando a média per capita de $\mathrm{R} \$ 39,01$ em 2010, Tabela 4.

O município de Aracitaba, em 2005, apresentou o menor valor de PRP com 0,62\%, enquanto Santa Margarida apresentou o valor máximo de 3,27\%. Em 2010 o município de Rosário de Limeira apresentou o menor valor de PRP com $0,72 \%$, enquanto o município de Rio Pomba com 6,44\%, apresentou o valor máximo.

O município que apresentou o menor valor de carga tributária per capita em 2005 foi Cipotânea com o valor de $\mathrm{R} \$ 9,57$ e o município de Sem Peixe apresentou valor máximo de $\mathrm{R} \$ 26,82$. Em 2010 o município de Rosário de Limeira atingiu o menor valor da carga tributária de $\mathrm{R} \$ 16,87$ e o município de Paiva apresentou o valor máximo.

Comparando os dados das tabelas nos anos analisados foi possível identificar um aumento da média da participação da receita própria em $44 \%$, podendo ser avaliado como um possível empenho dos municípios no aumento da arrecadação. $\mathrm{O}$ aumento da arrecadação própria é acompanhado pela ascensão em nível bem superior da carga tributária per capita, passando a população a pagar $\mathrm{R} \$ 20,51$ a mais em 2010 , representado um aumento de $110,86 \%$, o que pode ter afetado a renda da população.

\subsubsection{CLUSTER 2: ALTA PARTICIPAÇÃO DAS RECEITAS PRÓPRIAS E DA CARGA TRIBUTÁRIA}

Os municípios que compõe o Cluster 2 são caracterizados pela maior arrecadação própria entre os grupos em análise apresentando alto esforço fiscal. No entanto, é o grupo que também possui a maior carga tributária com os municípios sustentando a arrecadação própria por meio da elevação da carga tributária.

O Cluster 2 contém 4 observações (3,13\% do total examinado) em 2005 ampliando para 6 observações (4,69\% do total examinado) em 2010. Esse grupo não é composto pelos mesmos municípios nos dois períodos analisados, com exceção de Matias Barbosa. Os municípios de Itamarati de Minas, Ponte Nova e Santa Cruz do Escalvado. (75\% das observações de 2005) classificados no Cluster em 2005 passaram a compor o agrupamento 4 
Revista Ambiente Contábil - ISSN 2176-9036 - UFRN - Natal-RN. v. 8. n. 1, p. 1 - 16, jan./jun. 2016.

em 2010. Em contraponto, os municípios de Além Paraíba, Ewbank da Câmara, Santos Dumont, Sem Peixe e Viçosa passaram a fazer parte deste agrupamento em 2010.

A análise descritiva do Cluster 2 no período de 2005 e 2010 é apresentada na Tabela 5. A participação das receitas próprias dos municípios que compõem o Cluster 2 em 2005, variou de $5,91 \%$ a $8,60 \%$, apresentando média de 7,32\%. O indicador carga tributária per capita de impostos variou de $\mathrm{R} \$ 93,99$ a $\mathrm{R} \$ 113,53$ no ano de 2005 , apresentando uma média de $\mathrm{R} \$ 103,38$.

Conforme observado na Tabela 5, a participação da receita de tributos em 2010 variou de $6,96 \%$ a 15,74\%, apresentando média de 11,26\% em 2010. O indicador carga tributária per capita variou de $\mathrm{R} \$ 149,18$ a $\mathrm{R} \$ 216,81$, apresentando média de $\mathrm{R} \$ 181,65$ em 2010.

Tabela 5 - Análise descritiva Cluster 2

\begin{tabular}{ccccc}
\hline & PRP2005 $(\%)$ & PRP2010 $(\%)$ & CTIM2005 (R\$) & CTIM2010 (R\$) \\
\hline Média & 7,32 & 11,26 & 103,38 & 181,65 \\
\hline Mediana & 7,38 & 10,31 & 102,99 & 181,86 \\
\hline Moda & 5,91 & 6,96 & 93,99 & 149,18 \\
\hline Desvio padrão & 1,17 & 3,60 & 8,50 & 28,96 \\
\hline Variância & 1,36 & 12,99 & 72,31 & 838,53 \\
\hline Mínimo & 5,91 & 6,96 & 93,99 & 149,18 \\
\hline Máximo & 8,60 & 15,74 & 113,53 & 216,81 \\
\hline
\end{tabular}

Fonte: Dados da pesquisa.

O município de Itamarati de Minas apresentou em 2005 o menor valor de PRP de $5,91 \%$, enquanto Matias Barbosa com 8,60\%, apresentou o valor máximo. Em 2010 o município que apresentou o menor valor de PRP foi Matias Barbosa com 6,96\%, e o valor máximo de $15,74 \%$ foi apresentado no município de Santos Dumont.

O município de Matias Barbosa presente nos dois períodos apresentou queda de 19,07\% em 2010 na participação da receita de impostos em comparação a 2005, indicando baixo empenho dos municípios no estímulo ao aumento da arrecadação entre os períodos analisados.

$\mathrm{O}$ indicador carga tributária de impostos variou de $\mathrm{R} \$ 149,18$ a $\mathrm{R} \$ 216,81$, apresentando a média de $\mathrm{R} \$ 181,65$ em 2010. O município com menor valor de carga tributária em 2005 foi Ponte Nova com $\mathrm{R} \$ 93,99$ e de maior valor foi Matias Barbosa com $\mathrm{R} \$ 113,53$. Em 2010 o município que atingiu o menor valor da carga tributária foi Viçosa com $\mathrm{R} \$ 149,18$ e o município de Sem Peixe atingiu o maior valor de $\mathrm{R} \$ 216,81$.

Embora o número de municípios observados seja pequeno, este Cluster incluiu o município de Ponte Nova em 2005 e Viçosa em 2010. Estes municípios pertencem a subdivisão da microrregião da amostra analisada e destacam-se por apresentarem população maior em relação aos demais.

Analisando o Cluster identifica-se o aumento da média do indicador PRP em 53\% de 2005 para 2010, considerado avaliação positiva para os municípios que compõem o Cluster. Entretanto, o indicador CTIM aumentou sua média em $\mathrm{R} \$ 78,27$, apresentando $75 \%$ a mais quando comparado a 2005, indicando possível melhoria na gestão da arrecadação própria.

\subsubsection{CLUSTER 3: GESTÃO TRIBUTÁRIA PRÓPRIA INSTÁVEL}

O Cluster três apresentou comportamento mais instável quando comparado aos outros grupos. Em 2005 apresentou 36 observações $(28,13 \%$ do total examinado) e passou a ter apenas 1 observação (0,78\% do total examinado) em 2010. No ano de 2005 representava um grupo com esforço fiscal mediano, entretanto, em 2010, ao ser formado apenas por 1 município se caracteriza por uma alta arrecadação própria e elevada carga tributária per capita. Cabe destacar que na análise exploratória dos dados esse município não se apresentou como outliers. 
O grupo não é composto pelos mesmos municípios nos dois períodos analisados, com exceção do município de Santo Antônio do Grama (2,78\% das observações de 2005). Os 25 municípios que faziam parte desse Cluster em 2005 (69,44\% das observações de 2005) foram excluídos do grupo em 2010 e passaram a fazer parte do Cluster 1. Fazem parte desta transição os municípios de Acaiaca, Alto Caparaó, Antônio Prado de Minas, Astolfo Dutra, Carangola, Dom Silvério, Ervália, Guarani, Guarará, Lajinha, Lamim, Lima Duarte, Manhumirim, Paiva, Piau, Raul Soares, Recreio, Rio Casca, Rio Pomba, Rochedo de Minas, São Francisco do Glória, Senador Cortes, Silverânia, Tabuleiro e Tocantins.

Apenas o município de Ewbank da Câmara (2,78\% das observações de 2005) migrou para o grupo 2. Os municípios de Coronel Pacheco, Estrela Dalva, Goianá, Leopoldina, Pedra Dourada, Pequeri, Pirapetinga, Rio Preto e Volta Grande, que representaram 25\% das observações de 2005, migraram para o Cluster 4, totalizando uma saída de 35 municípios do Cluster 3 para os demais agrupamentos.

Pela análise descritiva do Cluster 3 no período de 2005, conforme Tabela 6, foi possível identificar que a variável participação das receitas de impostos dos municípios do Cluster 3 no ano de 2005 variou de $1,10 \%$ a 6,74\%, apresentando média de PRP de 3,07\%. O indicador carga tributária per capita de impostos em 2005 variou de $\mathrm{R} \$ 28,15$ a $\mathrm{R} \$ 49,32$, com a média de $\mathrm{R} \$ 35,87$.

Tabela 6 - Análise descritiva Cluster 3

\begin{tabular}{ccc}
\hline & PRP2005 (\%) & CTIM2005 (R\$) \\
\hline Média & 3,07 & 35,87 \\
\hline Mediana & 2,81 & 34,61 \\
\hline Moda & 1,10 & 28,15 \\
\hline Desvio padrão & 1,39 & 5,80 \\
\hline Variância & 1,93 & 33,66 \\
\hline Mínimo & 1,10 & 28,15 \\
\hline Máximo & 6,74 & 49,32 \\
\hline
\end{tabular}

Fonte: Dados da pesquisa.

Conforme exposto, em 2010, o Cluster foi composto por apenas 1 município, Santo Antônio de Grama, com PRP de 16,55\% e carga tributária per capita de R $\$ 371,14$. O município que teve em 2005 o menor valor de PRP foi Rochedo de Minas com 1,10\%, enquanto o que apresentou valor máximo foi Leopoldina 6,74\%.

\subsubsection{CLUSTER 4: REDUÇÃO DA PARTICIPAÇÃO DA ARRECADAÇÃO PRÓPRIA E AUMENTO DA CARGA TRIBUTÁRIA MUNICIPAL}

O Cluster 4 agrupou municípios com PRP e CTIM intermediária entre os grupos analisados. No entanto, foi o único grupo com o comportamento peculiar em que a PRP teve redução entre os períodos analisados. Ressalta-se que essa redução foi acompanhada de aumento significativo de $47 \%$ na carga tributária, representando queda na qualidade da gestão tributária em termos de arrecadação própria.

O grupo apresentava 13 observações $(10,15 \%$ do total dos municípios) em 2005 e passou a ter 24 observações (18,75\% do total dos municípios) em 2010. Esse Cluster não é composto pelos mesmos municípios nos dois períodos analisados, apenas 6 municípios permaneceram em ambos os anos.

Os municípios de Caparaó, Chácara, Mirai e Santa Bárbara do Monte Verde, representando 30,77\% das observações de 2005, que faziam parte desse Cluster em 2005 fora reclassificados para o Cluster 1 em 2010. Os municípios de Além Paraíba, Santos Dumont e Viçosa, que apresentaram 23,08\% das observações de 2005, foram reclassificados no Cluster 2. Um total de 7 municípios saíram deste Cluster no ano de 2005, sendo que os municípios de Coronel Pacheco, Estrela Dalva, Faria Lemos, Goiana, Itamarati de Minas, Laranjal, 
Revista Ambiente Contábil - ISSN 2176-9036 - UFRN - Natal-RN. v. 8. n. 1, p. 1 - 16, jan./jun. 2016.

Leopoldina, Pedra Bonita, Pedra Dourada, Pedro Teixeira, Pequeri, Piedade de Ponte Nova, Pirapetinga, Ponte Nova, Rio Preto, Santa Cruz do Escalvado, Tombos e Volta Grande passaram a fazer parte deste agrupamento em 2010.

Conforme observado na Tabela 7 , a variável participação da receita de impostos variou de 2,01\% a 8,56\% no ano de 2005, com a média de 6,49\% em 2005. Enquanto a variável carga tributária de impostos municipais per capita variou de $\mathrm{R} \$ 50,79$ a $\mathrm{R} \$ 81,57$ com média de $\mathrm{R} \$ 64,32$. Identificou-se que a participação das receitas próprias per capita dos municípios do Cluster 4 no ano de 2010 variou de 2,31\% a 9,71\% com média de 5,46\% em 2010. O indicador carga tributária de impostos variou de $\mathrm{R} \$ 68,84$ a $\mathrm{R} \$ 135,28$, com a média de $\mathrm{R} \$ 94,72$ em 2010.

Tabela 7 - Análise descritiva Cluster 4

\begin{tabular}{ccccc}
\hline & PRP2005 (\%) & PRP2010 $(\%)$ & CTIM2005 (R\$) & CTIM2010 (R\$) \\
\hline Média & 6,49 & 5,46 & 64,32 & 94,72 \\
\hline Mediana & 7,03 & 5,40 & 64,94 & 93,03 \\
\hline Moda & 2,01 & 2,13 & 50,79 & 68,84 \\
\hline Desvio padrão & 1,92 & 1,82 & 9,83 & 22,09 \\
\hline Variância & 3,67 & 3,31 & 96,56 & 487,83 \\
\hline Mínimo & 2,01 & 2,13 & 50,79 & 68,84 \\
\hline Máximo & 8,56 & 9,71 & 81,57 & 135,28 \\
\hline Fonte: Dados da pesquisa. & & & &
\end{tabular}

O município que apresentou em 2005 o menor valor de PRP foi Chácara com 2,01\%, enquanto de Santo Dumont apresentou o valor máximo de 8,56\%. Em 2010 o município que teve o menor valor de PRP foi Pedro Teixeira com 2,13\% e o maior valor foi apresentado pelo município de Cataguases com 9,71\%.

Em relação ao indicador carga tributária per capita o município apresentou a menor média em 2005 foi São João Nepomuceno com R \$50,79 e o de maior média apresentada foi o município de Caparaó com $\mathrm{R} \$ 81,57$. Em 2010 a menor média foi encontrada no município de Leopoldina com R $\$ 68,84$ e a maior valor de média foi alcançada pelo município de Coronel Pacheco com $\mathrm{R} \$ 135,28$.

Os municípios constantes no Cluster 4 apresentaram uma queda média da participação da receita de impostos de $15 \%$ em 2010 em relação a 2005. Este fato tende a ser negativo para os municípios que apresentam maior dependência nas transferências intergovernamentais. Ressalta-se um aumento da média de carga tributária per capita de $\mathrm{R} \$ 30,40$ (47\%) em 2010 em comparação a 2005.

\subsection{TESTE DE MÉDIAS PARA AMOSTRAS EMPARELHADAS}

Para realização do teste t para amostras emparelhadas comparou-se as variáveis de gestão tributária utilizadas neste estudo nos períodos de 2005 e 2010 . O intervalo de confiança adotado para tomada de decisão foi de $5 \%$.

Observa-se pela Tabela 8 , que demonstra os resultados para o teste $t$ em relação às variáveis PRP e CTIM, o valor da significância estatística de 0,00 está abaixo do intervalo de confiança tolerável de 0,05 . Dessa forma, indica-se a rejeição de $\mathrm{H}_{0}$ para as duas variáveis testadas, indicando a existência de evidências estatísticas suficientes para inferir sobre a diferença entre as médias das variáveis PRP e CTIM entre os dois períodos de análise, aceitando a hipótese $H_{A}$, ou seja, existe diferença na média da Participação dos Impostos na Receita Orçamentária e na média da Carga Tributária nos períodos de 2005 e 2010. 
Revista Ambiente Contábil - ISSN 2176-9036 - UFRN - Natal-RN. v. 8. n. 1, p. 1 - 16, jan./jun. 2016.

Tabela 8 - Resultados do Teste t para amostras emparelhadas

\begin{tabular}{|c|c|c|c|c|c|c|c|c|}
\hline & \multicolumn{4}{|c|}{ Diferenças emparelhadas } & \multirow[t]{3}{*}{$\mathrm{T}$} & \multirow[t]{3}{*}{$\mathrm{df}$} & \multirow{3}{*}{$\begin{array}{c}\text { Sig. (2 } \\
\text { extremidades) }\end{array}$} \\
\hline & & \multirow[t]{2}{*}{ Média } & \multirow[t]{2}{*}{$\begin{array}{l}\text { Desvio } \\
\text { padrão }\end{array}$} & \multicolumn{2}{|c|}{$\begin{array}{c}95 \% \text { Intervalo de } \\
\text { confiança da diferença }\end{array}$} & & & \\
\hline & & & & Inferior & Superior & & & \\
\hline Par 1 & $\begin{array}{l}\text { PRP2005 - } \\
\text { PRP2010 }\end{array}$ & $-0,830$ & 2,103 & $-1,198$ & $-0,462$ & $-4,466$ & 127 & 0,000 \\
\hline Par 2 & $\begin{array}{l}\text { CTIM2005 - } \\
\text { CTIM2010 }\end{array}$ & $-28,045$ & 42,143 & $-35,416$ & $-20,675$ & $-7,529$ & 127 & 0,000 \\
\hline
\end{tabular}

Fonte: Dados da pesquisa, 2014.

O valor negativo do Teste $t$ indica que a média do segundo grupo relativa a 2010 é maior que a do primeiro grupo, 2005. Dessa forma, pode-se afirmar o aumento na participação da arrecadação própria na receita orçamentária que foi acompanhado pelo aumento na carga tributária.

\section{CONSIDERAÇÕES FINAIS}

O objetivo deste estudo foi identificar a evolução da arrecadação tributária dos municípios da Zona da Mata Mineira entre os períodos de 2005 e 2010. De maneira geral os resultados apontam uma mudança positiva no perfil da arrecadação própria dos municípios entre os períodos analisados associado aos aumentos na carga tributária.

Observou-se que mesmo no Cluster 1, que agregou os municípios com baixo esforço fiscal, um aumento significativo na média da arrecadação própria entre os períodos analisados.

O comportamento inesperado dos municípios do Cluster 4 pode ser associado a aumentos no volume de transferências governamentais que implica no aumento da receita disponível e reduz consequentemente a participação da arrecadação própria na receita orçamentária total.

O teste de médias realizado comprovou estatisticamente a diferença nas médias da arrecadação própria, medida pela variável PRP, e da carga tributária, medida pela variável CTIM. O resultado do teste ainda apontou para aumento na média da arrecadação própria.

Em síntese, apesar da dependência dos recursos de transferências intergovernamentais, observou-se que os municípios têm realizados esforços para aumentar a arrecadação própria e consequentemente suas receitas disponíveis.

\section{REFERÊNCIAS}

ABRUCIO, F. L. A dinâmica federativa da educação brasileira: diagnóstico e propostas de aperfeiçoamento. In: Educação e federalismo no Brasil: combater as desigualdades, garantir a diversidade. (ORGs) OLIVEIRA, R. P., SANTANA, W. Brasília: UNESCO, 2010.

AFONSO, J. R. R.; ARAÚJO, É. A. A capacidade de gasto dos municípios brasileiros: arrecadação própria e receita disponível, BNDES, 2001.

ALCÂNTARA, F. H. C. As Reformas Legais e o Processo de Descentralização: Aspectos Jurídicos e Políticos. Revista Sociologia Política, Curitiba, v.19, n.39, p. 197-210, 2011.

ANDRADE, N. A. Contabilidade pública na gestão municipal: métodos com base na LC n. 101/00 e nas classificações contábeis advindas da SOF e STN.São Paulo: Atlas, 2010. 
BAIÃO, A. L.; CUNHA, A. S. M. da; SOUZA, S. R. N. de. Papel das Transferências Intergovernamentais na Equalizacão Fiscal dos Municípios Brasileiros. In. XXXVIII Encontro Nacional da Associação de Pós-Graduação em Administração - Enapad, Anais... Rio de Janeiro, 2014.

BOVO, J. M. Gastos sociais dos municípios e desequilíbrio financeiro. Revista de Administração Pública, v. 35, n. 1, p. 93-117, 2001.

CORRAR, L. J.; PAULO, E.; DIAS FILHO, J. M. Análise multivariada. São Paulo: Atlas, 2007.

GOMES, G. M.; MAC DOWELL, M. C. Descentralização Política, Federalismo Fiscal e Criação de Municípios: O que é Mau para o Econômico nem sempre é Bom para o Social. Texto para Discussão nº 706. Brasília: IPEA, 2000.

GOUVÊA, M. A.; VARELA, P. S.; FARINA, M. C. Avaliação das relações entre receita tributária e desenvolvimento econômico e social dos grupos 3, 4 e 5 de municípios paulistas, segundo o IPRS: uso da análise multivariada de variância. Revista Eletrônica de Administração, v. 16, n. 2, p. 1-21, 2010.

KOHAMA, H. Balanços públicos: teoria e prática. São Paulo: Atlas, 1999.

LINHARES, P. T. F.; MENDES, C. C.; LASSANCE, A. Federalismo à brasileira: questões para discussão. Brasília: Ipea, 2012.

MASSARDI, W. O. ABRANTES, L. A. Classificação dos Municípios Mineiros em Relação à Composição de suas Receitas. Revista de Gestão, Finanças e Contabilidade, Salvador, v. 4, n. 1, p. 144-161, 2014.

MATIAS, A. B.; CAMPELLO, C. A. G. Administração financeira municipal. Atlas, 2000.

MATIAS-PEREIRA, J. Finanças Públicas. A política orçamentária no Brasil. $4^{\text {a }}$ edição. São Paulo: Editora Atlas, 2009.

MENDES, M., MIRANDA, R. B.; COSSIO, F. (2008). Transferências intergovernamentais no Brasil: diagnóstico e proposta de reforma, Consultoria Legislativa do Senado Federal, Texto para Discussão 40, Abril.

OLIVEIRA, E. J.; BARBOSA, J. L.; FRANÇA, M. A Reforma do Estado Brasileiro e a Descentralização: Implicações do Federalismo Fiscal nos Estados e Municípios. Revista Educação e Políticas em Debate, v. 2, n. 2, p. 405-416, 2013.

REIS, P. R. da C.; COSTA, T. de M. T. da; SILVEIRA, S. F. R. Receita Pública e Bem-Estar Social nos Municípios Mineiros Emancipados no Período de 1988 A 1997. Revista Eletrônica de Administração, v. 19, n. 1, p. 61-82, 2013.

REZENDE, F. Federalismo fiscal: em busca de um novo modelo. In: Educação e federalismo no Brasil: combater as desigualdades, garantir a diversidade. (ORGs) 
OLIVEIRA, R. P.; SANTANA, W. Brasília: UNESCO, 2010.

Os Desafios do Federalismo Fiscal. In: REZENDE, F.. (Org.). Desafios do Federalismo Fiscal. Rio de Janeiro: FGV Editora, 2006.

SERRA, J.; AFONSO, J. R. R. Federalismo Fiscal à Brasileira: Algumas Reflexões. Revista do BNDES, Rio de Janeiro, v.6, n.12, p. 3-30, dez. 1999.

SOUZA, C. Federalismo e conflitos distributivos: disputa dos estados por recursos orçamentários federais. DADOS. Revista de Ciências Sociais, Rio de Janeiro, v. 46, n. 2, p. 345-84, 2003.

TOMIO, F. R. de L. Federalismo, Municípios e Decisões e Legislativas: A Criação de Municípios no Rio Grande do sul. Revista Sociologia Política, Curitiba, 24, p. 123-148, 2005 . 